\title{
Genetic dissection of plant growth habit in chickpea
}

\author{
Hari D. Upadhyaya ${ }^{1} \cdot$ Deepak Bajaj $^{2} \cdot$ Rishi Srivastava $^{2} \cdot$ Anurag Daware $^{2}$. \\ Udita Basu $^{2}$ • Shailesh Tripathi ${ }^{3}$. Chellapilla Bharadwaj ${ }^{3}$. Akhilesh K. Tyagi ${ }^{2,4}$. \\ Swarup K. Parida ${ }^{2}$
}

Received: 11 April 2016/Revised: 1 May 2017 / Accepted: 26 May 2017

(C) Springer-Verlag Berlin Heidelberg 2017

\begin{abstract}
A combinatorial genomics-assisted breeding strategy encompassing association analysis, genetic mapping and expression profiling is found most promising for quantitative dissection of complex traits in crop plants. The present study employed GWAS (genome-wide association study) using 24,405 SNPs (single nucleotide polymorphisms) obtained with genotyping-by-sequencing (GBS) of 92 sequenced desi and kabuli accessions of chickpea. This identified eight significant genomic loci associated with erect (E)/semi-erect (SE) vs. spreading (S)/semi-spreading (SS)/prostrate $(\mathrm{P})$ plant growth habit $(\mathrm{PGH})$ trait differentiation regardless of diverse desi and kabuli genetic backgrounds of chickpea. These associated SNPs in combination explained 23.8\% phenotypic variation for PGH in chickpea. Five PGH-associated genes were validated successfully in E/SE and SS/S/P PGH-bearing parental accessions and homozygous individuals of three intra-
\end{abstract}

Hari D. Upadhyaya and Deepak Bajaj contributed equally to this work.

Electronic supplementary material The online version of this article (doi:10.1007/s10142-017-0566-8) contains supplementary material, which is available to authorized users.

Swarup K. Parida

swarup@nipgr.ac.in; swarupdbt@gmail.com

1 International Crops Research Institute for the Semi-Arid Tropics (ICRISAT), Patancheru, Telangana 502324, India

2 National Institute of Plant Genome Research (NIPGR), Aruna Asaf Ali Marg, New Delhi 110067, India

3 Division of Genetics, Indian Agricultural Research Institute (IARI), New Delhi 110012, India

4 Present address: Department of Plant Molecular Biology, University of Delhi South Campus, New Delhi 110021, India and interspecific RIL (recombinant inbred line) mapping populations as well as 12 contrasting desi and kabuli chickpea germplasm accessions by selective genotyping through Sequenom MassARRAY. The shoot apical, inflorescence and floral meristems-specific expression, including upregulation (seven-fold) of five PGH-associated genes especially in germplasm accessions and homozygous RIL mapping individuals contrasting with E/SE PGH traits was apparent. Collectively, this integrated genomic strategy delineated diverse non-synonymous SNPs from five candidate genes with strong allelic effects on PGH trait variation in chickpea. Of these, two vernalization-responsive non-synonymous SNP alleles carrying SNF2 protein-coding gene and B3 transcription factor associated with PGH traits were found to be the most promising in chickpea. The SNP allelic variants associated with $\mathrm{E} / \mathrm{SE} / \mathrm{SS} / \mathrm{S} \mathrm{PGH}$ trait differentiation were exclusively present in all cultivated desi and kabuli chickpea accessions while wild species/accessions belonging to primary, secondary and tertiary gene pools mostly contained prostrate PGHassociated SNP alleles. This indicates strong adaptive natural/ artificial selection pressure (Tajima's D 3.15 to 4.57) on PGHassociated target genomic loci during chickpea domestication. These vital leads thus have potential to decipher complex transcriptional regulatory gene function of PGH trait differentiation and for understanding the selective sweep-based PGH trait evolution and domestication pattern in cultivated and wild chickpea accessions adapted to diverse agroclimatic conditions. Collectively, the essential inputs generated will be of profound use in marker-assisted genetic enhancement to develop cultivars with desirable plant architecture of erect growth habit types in chickpea.

Keywords Chickpea - GWAS · Plant growth habit · QTL · SNP 


\section{Introduction}

Chickpea (Cicer arietinum L.) is a diploid annually grown self-pollinated food legume crop with a high economic value having a genome size of $\sim 740 \mathrm{Mbp}$ and known to be nutritionally rich (Kumar et al. 2011; Gaur et al. 2012). The cultivated chickpea (desi and kabuli) representing diverse genepools with contrasting agronomic traits believed to have originated from its wild $C$. reticulatum progenitor for all of which the draft genomes have been sequenced recently (Jain et al. 2013; Varshney et al. 2013, 2014; Parween et al. 2015; Gupta et al. 2016). The cultivated desi and kabuli chickpea usually exhibit erect to semi-erect plant growth habit while accessions belonging to wild species commonly have prostrate growth habit. This indicates that plant growth habit could be a common target trait for both domestication and artificial breeding in chickpea (Wang and Li 2008; Meyer et al. 2012).

The plant growth habit is a vital agronomic trait in chickpea that varies widely among desi, kabuli and wild accessions (Upadhyaya et al. 2006). The chickpea germplasm accessions are primarily classified and characterized into five plant growth habit types - erect $\left(0-15^{\circ}\right.$ angle of primary branches from the vertical axis at mid-pod filling stage), semi-erect (16$\left.25^{\circ}\right)$, semi-spreading $\left(26-60^{\circ}\right)$, spreading $\left(61-80^{\circ}\right)$ and prostrate (branches creeping above the ground) (Upadhyaya et al. 2008). The growth habit is a crucial determinant of plant architecture and plays a key role in influencing various seed and pod yield component traits in chickpea as well as adaptation of its plant-type to diverse agroecological environments. The plant growth habit is thus a complex yield contributing quantitative trait and usually governed by convoluted interplay of multiple genes/QTLs (quantitative trait loci) especially regulating diverse developmental processes in chickpea (Benlloch et al. 2015).

Considering the agronomic importance of growth habit in defining ideal plant architecture and seed/pod yield enhancement as well as affecting plant adaption to its environment, it is imperative to dissect the genetic and molecular basis underlying this broad trait variation existing among natural germplasm accessions of chickpea (Wang and Li 2008; Meyer et al. 2012). Attempts have been made to develop improved cultivars with desirable plant type by exploiting diverse potential wild Cicer species accessions in introgression breeding program of chickpea genetic enhancement (Cobos et al. 2009; Aryamanesh et al. 2010; Benlloch et al. 2015). However, the genetic drag of undesirable traits like spreading/prostrate growth habit and late flowering is the major hindrance to such process of conventional introgression breeding for chickpea genetic improvement. To substantiate these efforts, genomicsassisted breeding strategy by molecular mapping of major QTLs predominantly associated with erect/semi-erect and prostrate growth habits on multiple interspecific genetic linkage maps $(C$. arietinum $\times C$. reticulatum $)$ have been deployed to understand the complex genetic architecture and inheritance pattern of plant growth habit trait variation in chickpea (Cobos et al. 2009; Aryamanesh et al. 2010). For instance, a candidate genetic locus $(H g / h g)$ underlying a major erect/prostrate QTL has been mapped on the linkage group 3 of an interspecific genetic map (semi-erect C. arietinum desi cv. ICCL $81001 \times$ prostrate C. reticulatum wild cv. Cr5-9) (Cobos et al. 2009). Alongside, another candidate genetic locus (Prostrate) harbouring a major prostrate QTL has been mapped on the linkage group 3 of an interspecific genetic map (semi-erect C. arietinum desi $\mathrm{cv}$. ICC $3996 \times$ C. reticulatum wild $\mathrm{cv}$. ILWC 184) (Aryamanesh et al. 2010). These studies infer that the genetic analysis of plant growth habit trait is mostly limited to estimation of heritability and genetic mapping of few long marker interval-spanning major growth habit QTLs due to their quantitative and polygenic nature of inheritance in chickpea (Cobos et al. 2009; Aryamanesh et al. 2010). However, these major QTLs governing growth habit are yet to be validated across diverse genetic backgrounds and environments prior to their deployment in fine-mapping/mapbased gene isolation and marker-assisted breeding program for developing improved cultivars of desirable plant type in chickpea. In this context, comprehensive understanding on complex genetic inheritance pattern by quantitative dissection of plant growth habit is essential to decipher the regulatory function and domestication pattern of genes/QTLs controlling these traits in chickpea.

Considering the efficacy of integrated genomics-assisted breeding strategy (association study, QTL mapping and expression profiling) for genetic dissection of various complex quantitative traits in crop plants, this approach can be deployed in large-scale natural and mapping populations to delineate functionally relevant potential molecular tags (markers, genes/QTLs, alleles and haplotypes) regulating plant growth habit in chickpea (Bajaj et al. 2015a, b, 2016; Das et al. 2015, 2016; Kujur et al. 2015a, b; Upadhyaya et al. 2015, 2016). The aforesaid integrated strategy will also assist us in unravelling the molecular genetic basis of natural plant growth habit trait variation existing in various cultivated and wild accessions of chickpea adapted to diverse agroclimatic regions. The resultant findings on complex plant growth habit trait dissection can essentially be utilized in genomics-assisted breeding program to develop superior cultivars with preferred genetic attributes of erect plant type in chickpea. The development of cultivars with restructured erect plant type will not only facilitate higher pod/seed yield and mechanization of harvesting in chickpea but also mitigate the effects of acute climate change for sustaining global food security.

In light of the above, the present study employed an integrated genomics-assisted breeding strategy involving genome-wide association study (GWAS), selective genotyping and differential expression profiling to delineate functionally relevant natural allelic variants in potential candidate 
gene(s) regulating plant growth habit traits, with an eventual objective of developing ideal erect new plant type for accelerating chickpea genetic enhancement.

\section{Materials and methods}

\section{Phenotyping for plant growth habits}

A total of 92 including 39 desi and 53 kabuli chickpea accessions were grown in the field following randomised complete block design (RCBD) with at least two replications during normal crop season for two consecutive years (2012 and 2013) at two diverse geographical locations (Patancheru, Hyderabad: latitude $17^{\circ} 3^{\prime} \mathrm{N} /$ longitude $77^{\circ} 2^{\prime} \mathrm{E}$ and New Delhi: $28^{\circ} 4^{\prime} \mathrm{N} / 77^{\circ} 2^{\prime}$ E) of India (Table S1). In addition, 190 individuals representing each of two intraspecific $\mathrm{F}_{7}$ RIL (recombinant inbred lines) mapping populations: ICC 13523 (erect kabuli landrace) $\times$ ICC 12299 (semi-spreading desi landrace) and ICC 4958 (semi-erect desi landrace) $\times$ ICC 12299 as well as one interspecific $\mathrm{F}_{8}$ RIL mapping population: ICC 4958 (semi-erect desi landrace) $\times$ ICC 17160 (prostrate wild accession) were grown as per aforementioned strategies. The angle of primary branches from the vertical axis of each accession and RIL mapping individual (2-4 representative plants from each accession/individual) were measured at their mid-pod filling stage. The germplasm accessions and mapping individuals of chickpea were classified predominantly into diverse plant growth habit types, including erect (E), semi-erect (SE), semi-spreading (SS) and spreading (S) in accordance with the estimation of 0-15, 16-25, 26-60, 61$80^{\circ}$ angles of their primary branches, respectively, from the vertical while prostrate $(\mathrm{P})$ growth habit was measured based on flatness of branches over the ground.

The diverse statistical parameters, including coefficient of variation (CV), analysis of variance (ANOVA) and frequency distribution of diverse plant growth habit (PGH) traits phenotyped among accessions and RIL mapping individuals were estimated following Bajaj et al. (2015a). The inheritance pattern of PGH traits was determined by measuring the effect of genotypes (accessions) $(\mathrm{G})$, environments (experimental years and/or geographical locations) (E) and $\mathrm{G} \times \mathrm{E}$ interactions using ANOVA. The broad-sense heritability $\left[\mathrm{H}^{2}=\sigma^{2} \mathrm{~g} /\right.$ $\left.\left(\sigma^{2} \mathrm{~g}+\sigma^{2} \mathrm{ge} / \mathrm{n}+\sigma^{2} \mathrm{e} / \mathrm{nr}\right)\right]$ of PGH traits was measured employing optimum criteria including $\sigma^{2}$ g (genetic), $\sigma^{2}$ ge $(\mathrm{G} \times \mathrm{E})$ and $\sigma^{2} \mathrm{e}$ (error) variance with $n$ (number of experimental years/environments) $=2$ and $r$ (number of replicates) $=2$.

\section{Trait association mapping}

The genotyping data including structural/functional annotation information of kabuli reference genome- and de novo-based
SNPs mined from the sequenced 92 (39 desi and 53 kabuli) chickpea accessions using a GBS (genotyping-by-sequencing)assay were acquired (Kujur et al. 2015a, c). For GWAS of PGH traits, the genetic diversity information including phylogenetic tree, PCA (principal component analysis) and population structure among 92 chickpea accessions were obtained from our previous study (Kujur et al. 2015a, c). The population structure ancestry coefficient $(\mathrm{Q})$, kinship matrix $(\mathrm{K})$ and PCA $(\mathrm{P})$ data along with genome-wide SNP genotyping and PGH phenotyping information of 92 accessions were analysed through mixed model ( $\mathrm{P}+\mathrm{K}, \mathrm{K}$ and $\mathrm{Q}+\mathrm{K}$ )-based CMLM (compressed mixed linear model) and P3D (population parameters previously determined, Zhang et al. 2010; Kang et al. 2011)/EMMAX (efficient mixed model association eXpedited) approaches of GAPIT (Lipka et al. 2012) as described previously (Kujur et al. 2015a; Bajaj et al. 2016; Upadhyaya et al. 2015, 2016). To ascertain the accuracy and validity of SNP marker-trait association, the observed and expected $-\log _{10}(P)$ value relative distribution estimated for each PGH-associated genomic locus was compared based on quantile-quantile plot. Subsequently, the correction of their adjusted $P$ value threshold of significance for multiple comparison was performed by false discovery rate (FDR cut-off $\leq 0.05$, Benjamini and Hochberg 1995) following Kujur et al. (2015a), Upadhyaya et al. (2015, 2016) and Bajaj et al. (2016). The degree of association of SNP loci with diverse PGH traits was measured by the $R^{2}$ using a model with the SNPs and adjusted $P$ value adopting a FDR-controlling strategy. The SNP loci in the genomic/gene regions exhibiting significant association with PGH traits at the lowest FDR adjusted $P$ values (threshold $P<10^{-8}$ ) and highest $R^{2}$ (degree of SNP marker-trait association) were identified in chickpea.

\section{Validation of PGH-associated SNPs in natural and mapping populations}

To ensure the potential of detected genomic SNP loci for PGH trait association, these SNPs were targeted to validate among natural germplasm accessions as well as in two intraspecific $\mathrm{F}_{7}$ RIL mapping populations: (ICC $13523 \times$ ICC 12299) and (ICC $4958 \times$ ICC 12299) and one inter-specific $\mathrm{F}_{8}$ RIL mapping population: (ICC $4958 \times$ ICC 17160) contrasting with diverse PGH traits in chickpea. For this, three parental accessions and 10 of each homozygous RIL individuals of three aforesaid mapping populations along with 12 desi and kabuli germplasm accessions (selected from 92 accessions used for GWAS) exhibiting variations for diverse contrasting PGH traits (E, SE, SS, S and P) were selected for genomic DNA isolation. The PGH-associated SNPs showing polymorphism between the parents of three mapping populations were further genotyped in the aforementioned homozygous mapping individuals and germplasm accessions using MALDI-TOF MS (matrix-assisted laser desorption/ionization time-of-flight mass spectrometry) SNP genotyping assay as per Saxena et al. (2014a, b). The PGH-associated SNP allelic 
variants (scanned by GWAS) identified from homozygous accessions/individuals were correlated with diverse PGH traits to validate the trait association potential of target genomic loci. Furthermore, the physical positions (bp) of PGH-associated SNPs localized on chromosomes were correlated with that of multiple known major PGH QTLs mapped earlier on the interspecific genetic linkage maps to determine the validity and novelty of these GWAS-derived major genomic loci identified in chickpea.

\section{Differential expression profiling}

To determine the expression pattern of candidate genes ensured by GWAS and selective genotyping, the differential expression profiling of PGH-associated genes was performed using the semi-quantitative and quantitative RT-PCR assays. The RNA was isolated from vegetative and reproductive tissues [leaf, root, shoot apical meristem (SAM), inflorescence and floral meristems] of parental accessions and two of each homozygous individuals derived from aforementioned three mapping populations as well as four desi and kabuli germplasm accessions contrasting with diverse (E/SE and P/SS/S) PGH traits (Table S2). The isolated RNA was amplified with the gene-specific primers and differential expression analysis was performed following Bajaj et al. (2015c) and Upadhyaya et al. (2015). RNA isolated from three independent biological replicates of each sample and two technical replicates of each biological replicate with no template and primer as control were used in the quantitative RT-PCR assay. One microgram of high quality total RNA was utilized for cDNA synthesis using first strand cDNA synthesis kit (Applied Biosystems, USA). The cDNA (1:100 dilution) along with 1X Fast SYBR Green Master Mix (Applied Biosystems) and $200 \mathrm{nM}$ of forward and reverse gene-specific primers in a total reaction volume of $10 \mu \mathrm{l}$ were amplified in 7500 Fast RealTime PCR system (Applied Biosystems). The differential expression profiles of genes among tissues/stages of germplasm accessions and RIL mapping individuals were determined as per Bajaj et al. (2015c) and Upadhyaya et al. (2015). An internal control gene elongation factor 1-alpha (EFl $\alpha)$ exhibiting consistent expression across diverse tissues and developmental stages of chickpea accessions/individuals was utilized for normalization of expression value. The significant difference in gene expression was determined by a LSD-ANOVA significance test using SPSS 17.0 (http://www.spss.com/statistics) and visualized with a heat map employing MultiExperiment Viewer (MeV, http://www. tm4.org/mev).

\section{Natural allelic diversity in PGH-associated candidate genes}

To determine natural allelic diversity, PGH-associated candidate genes validated by GWAS, selective genotyping and differential expression profiling were selected. The 100-bp sequence regions flanking the associated SNP loci were PCR amplified using the genomic DNA of 79 wild chickpea accessions (belonging to primary, secondary and tertiary gene pools) and 92 cultivated (desi and kabuli) chickpea accessions (selected for GWAS). A representative set of 79 wild accessions were selected from five annual wild species, namely, C. reticulatum (14 accessions), C. echinospermum (8), C. judaicum (22), C. bijugum (19) and C. pinnatifidum (15) belonging to primary and secondary gene pools, and one perennial species $C$. microphyllum of tertiary gene pool. The PCR amplicons were cloned and sequenced, and SNPs were mined among accessions following Kujur et al. (2013), Saxena et al. (2014a) and Upadhyaya et al. (2015). To estimate various nucleotide diversity measures such as average pairwise nucleotide diversity $(\theta \pi)$ and Watterson's estimator of segregating sites $(\theta \omega)$ and Tajima's $\mathrm{D}$, a 100-kb non-overlapping sliding window approach of TASSEL v5.0 (http://www. maizegenetics.net) was utilized as per Xu et al. (2011) and Kujur et al. (2015c).

\section{Results and discussion}

\section{GWAS of PGH traits in chickpea}

An optimized NGS-based GBS strategy was utilized to discover and genotype genome-wide SNPs and subsequently in GWAS for identification of potential genes and natural allelic variants regulating diverse PGH traits in chickpea. In the present study, 24,405 high-quality SNPs discovered from 92 sequenced desi and kabuli accessions employing reference genome (16376)- and de novo (8029)-based GBS assays were further integrated with replicated multilocation/year PGH field phenotyping information to perform GWAS of PGH traits in chickpea (Table S3). The high-quality 14,115 SNPs physically mapped on eight kabuli chickpea chromosomes were submitted to NCBI dbSNP (http://www.ncbi.nlm.nih. gov/SNP/snp_viewTable.cgi?handle=NIPGR) with SNP submission (SS) accession numbers $(2,728,910,337$ to $2,728,924,439)$ for unrestricted public access (Table S3). The aforesaid SNP genotyping information-led neighbourjoining phylogenetic tree, high-resolution population genetic structure and PCA differentiated all 92 accessions from each other and clustered into two distinct populations; POP I and POP II representing primarily the kabuli and desi chickpea cultivars with E, SE, S and SS plant growth habits. This agreed well with our previous studies that utilized genomewide GBS-derived SNPs for determining the evolutionary relationship and population genetic structure among a similar set of 92 desi and kabuli chickpea accessions (Kujur et al. 2015a, c). The normal frequency distribution along with a broader PGH phenotypic variation and $80 \%$ broad-sense heritability $\left(\mathrm{H}^{2}\right)$ among 92 chickpea accessions was evident. We utilized 
the field phenotyping data of accessions exclusively revealing consistent phenotypic expression (sustained by high $\mathrm{H}^{2}$ ) of PGH traits across two geographical locations/year for subsequent SNP marker-trait association analysis. These observations indicated that the 92 natural chickpea germplasm accessions representing 16 diverse ecogeographical regions of the world screened by us for GWAS are rich in allelic diversity for diverse PGH traits. Therefore, the selected accessions can serve as a useful genetic resource for mining novel functional allelic variants to expedite trait association mapping of PGH at a whole genome and/or gene-level in chickpea. This will essentially accelerate identification of potential genes and natural allelic variants governing diverse PGH traits at a genomewide scale in domesticated chickpea.

The CMLM and P3D/EMMAX model-based association mapping approach (FDR cut-off $\leq 0.05$ ) identified eight genomic loci (gene-based SNPs) exhibiting significant association $\left(P \leq 10^{-8}\right)$ with diverse PGH traits in chickpea (Fig. 1, Table 1). This included seven SNP loci which were physically mapped on six chromosomes. The remaining one SNP locus was represented from scaffold region of chickpea genome. Maximum number of three PGH-associated SNPs were mapped on chromosome 4 (Fig. 1, Table 1). One of eight PGH-associated genomic SNP loci was derived from the intergenic region; whereas, rest seven SNP loci were represented from diverse coding (six non-synonymous SNPs) and non-coding intronic (one SNP) sequence components of seven kabuli genes (Table 1). The detected eight significant SNPs explained $10-17 \%$ phenotypic variation for diverse PGH traits observed in 92 chickpea accessions. The total phenotypic variation for PGH traits explained by eight SNPs in combination was $23.8 \%$. Six SNP loci from the six candidate genes were significantly associated with E/SE vs. P/SS/S PGH trait differentiation in chickpea (Table 1). The remaining one of each intergenic and gene-derived SNP were identified to be associated with E/SE vs. SS/S PGH trait differentiation. The significant association of eight SNP loci mapped in more than one genomic regions (genes) with diverse PGH traits infers complex genetic inheritance pattern and regulation of these target quantitative traits in chickpea. A significant difference concerning the association potential of SNP loci with PGH traits in two different desi and kabuli populations could not be observed despite diverse genetic architecture of PGH traits in 92 accessions belonging to two populations (POP I and II) and along entire population. Henceforth, PGH-associated genomic loci (genes) identified by us employing GWAS have efficacy to be deployed in marker-aided genetic enhancement of chickpea. Remarkably, strong association of nonsynonymous coding SNPs-containing two genes, namely, major intrinsic protein (MIP) [glutamic acid (GAA) to glycine (GGA)] and sucrose non-fermenting (SNF2) [leucine (CTT) to phenylalanine (TTT)] with E/SE vs. P/SS/S PGH trait differentiation (P: $1.2-1.5 \times 10^{-10}$ with $15-17 \%$ PVE) as compared to that of other six associated SNPs was apparent (Table 1). The non-synonymous SNPs from the candidate genes associated with diverse PGH traits delineated in our study using GWAS have functional significance towards rapid dissection of these complex quantitative traits in chickpea. The non-synonymous SNPs in the genes are known to alter their transcriptional regulatory mechanism for controlling diverse yield and quality component traits in chickpea (Kujur et al. 2013, 2014; Bajaj et al. 2015a, b, c; Upadhyaya et al. 2015). Aside, the added advantages of large-scale SNPs discovered especially from the genes annotated on the chromosomes for high-resolution trait association mapping to delineate potential genes regulating vital agronomic traits have been well documented in crop plants with small diploid and large complex polyploid genomes (Akpinar et al. 2017). Therefore, clues obtained from our study further can essentially be employed for establishing rapid marker-trait linkages and efficient PGH trait-regulatory gene identification and characterisation in chickpea. The GWAS is a much proficient and attractive strategy for quantitative dissection of diverse complex agronomic traits in crop plants including chickpea

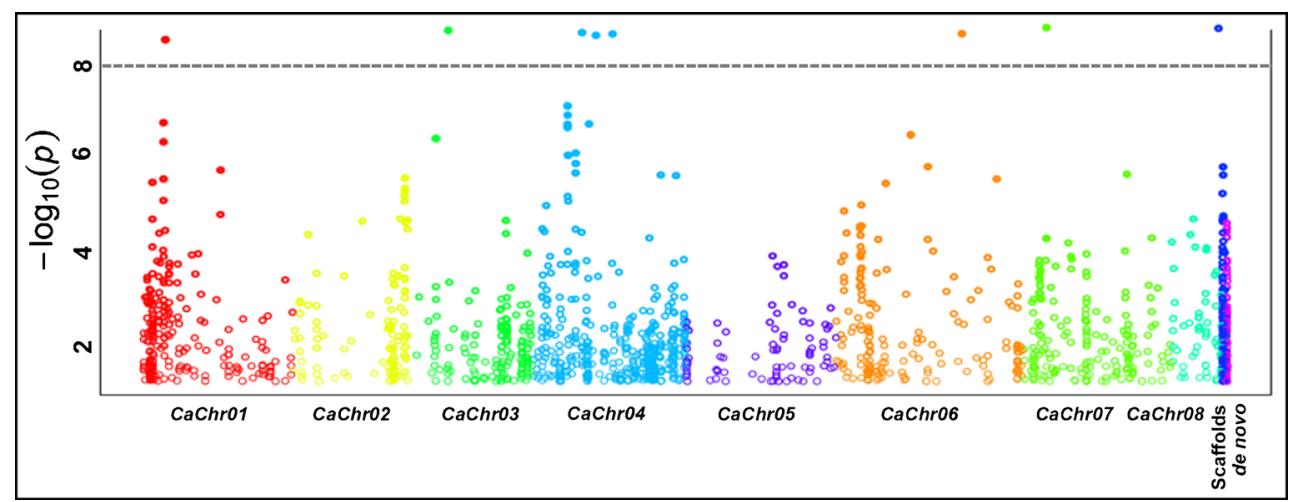

Fig. 1 GWAS-derived Manhattan plot illustrating significant (FDR cutoff $<0.05)-\log _{10}(P)$ values $(y$-axis) associated with $\mathrm{S} / \mathrm{SE}$ vs. P/SS/S PGH trait differentiation using 24,405 reference genome and de novo-based GBS-SNPs. The $x$-axis represents the genomic distribution of SNPs physically mapped on eight chromosomes and scaffolds of chickpea genome. The SNPs revealing significant association with diverse PGH traits at a cut-off $P$ value $\leq 1 \times 10^{-8}$ are marked with a dotted line 


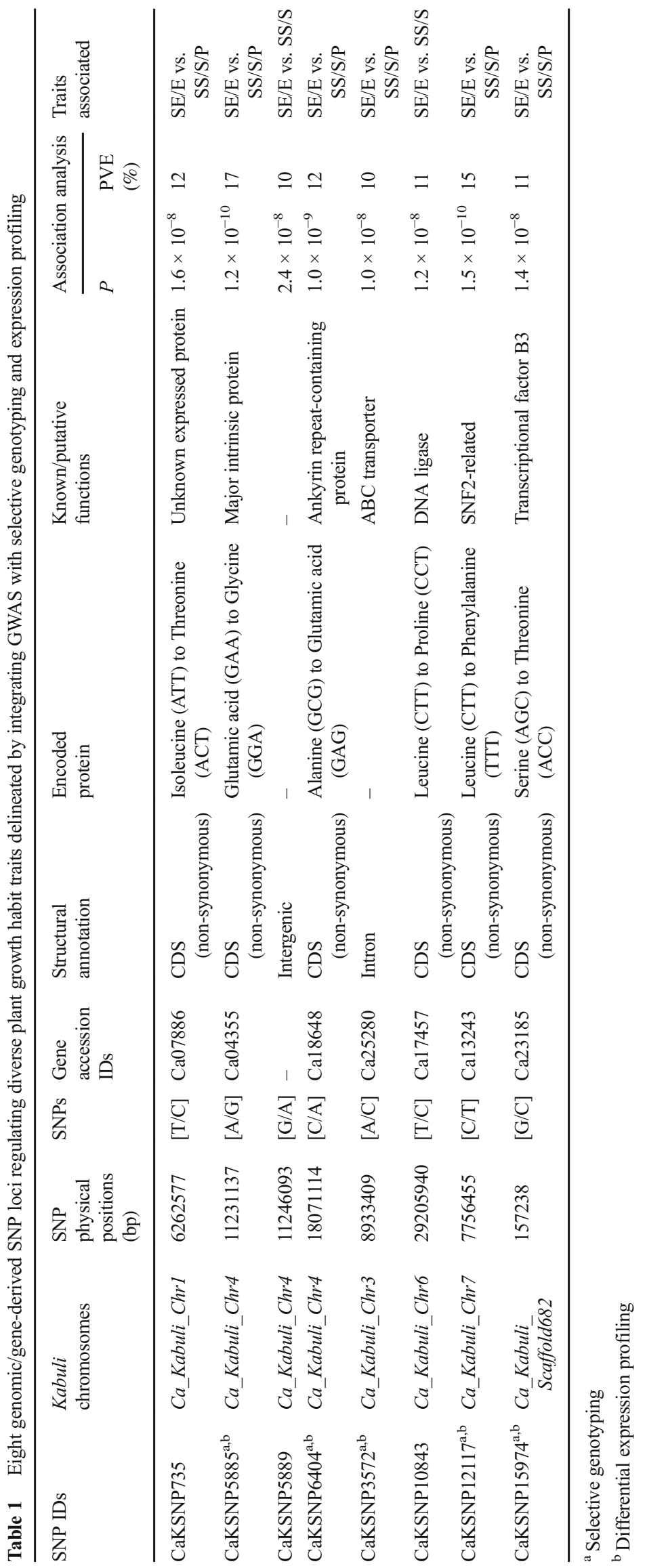


(Feuillet et al. 2012; Thudi et al. 2014; Kujur et al. 2015a; Upadhyaya et al. 2015, 2016; Bajaj et al. 2016; Liu et al. 2016). In this perspective, a high-resolution GWAS approach deployed by us to scale down the potential genomic loci/genes governing quantitative traits of PGH at a genome-wide scale appears much expedient for understanding the complex genetic architecture of target traits in order to drive genomicsassisted crop improvement of chickpea (Kujur et al. 2015a; Bajaj et al. 2016; Upadhyaya et al. 2015, 2016).

\section{Validation of PGH-associated genomic loci by selective genotyping in mapping and natural populations}

To validate the PGH-associated SNP loci, SNPs revealing parental polymorphism were genotyped in 10 of each homozygous individuals derived from two intraspecific [(ICC $13523 \times$ ICC 12299) and (ICC $4958 \times$ ICC 12299)] and one interspecific (ICC $4958 \times$ ICC 17160) RIL mapping populations as well as among 12 desi and kabuli germplasm accessions contrasting with E/SE and S/SS plant growth habit traits. Four non-synonymous SNPs and one intronic SNPcontaining candidate genes (major intrinsic protein, ankyrin repeat domain containing protein, $\mathrm{ABC}$ transporter, sucrose non-fermenting protein and $\mathrm{B} 3$ transcription factor) exhibiting significant association $\left(P=1.37 \times 10^{-8}\right)$ with $\mathrm{PGH}$ traits (based on our GWAS) were validated in both intra-and interspecific mapping populations contrasting for diverse PGH traits by selective genotyping (Figs. S1, 2, 3, Table 1). Interestingly, the occurrence of identical E/SE and S/SSassociated alleles derived from these five gene-based SNP loci in parental accessions (ICC 13523, ICC 12299, ICC 4958 and ICC 17160) and homozygous mapping individuals contrasting with $\mathrm{E} / \mathrm{SE}$ and $\mathrm{S} / \mathrm{SS} \mathrm{PGH}$ traits was observed (Figs. S1, 2, 3). Likewise, five gene-derived SNP alleles were validated in E/SE (ICC 16814, ICC 6306, ICC 7323, ICC 11284, ICC 1161, ICC 15061 and ICC 8261) and S/SS (ICC 5590, ICC 11584, ICC 1164, ICC 13044 and ICC 15888) PGH-bearing natural germplasm accessions (Figs. S1, 2, 3). In order to ascertain the accuracy/validity and novelty of five SNP locicarrying genes for PGH trait association (validated by GWAS and selective genotyping) in chickpea, the outcome of the current study was compared and correlated with that of previous studies on molecular mapping of known major PGH QTLs documented in this important food legume crop. To perform this, markers linked/flanking the known major PGH QTLs (reported by previous QTL mapping studies) were selected for their further validation in the PGH-specific 92 desi and kabuli natural chickpea germplasm accessions and three inter-/intra-specific mapping populations constituted in our study. This revealed correspondence of one intronic SNPcontaining gene (ABC transporter) harbouring the known major PGH (E/P) QTLs mapped on linkage group 3 between past and present studies based on congruent flanking/linked marker physical positions (bp) on the chickpea chromosomes (Cobos et al. 2009; Aryamanesh et al. 2010). In this context, seven novel and one previously documented SNPs-containing known/candidate genes governing diverse PGH traits delineated in our study could be considered as potential candidates for their utilization in marker-assisted genetic improvement with an ultimate objective of developing restructured ideal erect plant type in chickpea.

\section{Validation of PGH-associated genes through expression profiling}

The PGH-associated eight genes, including five validated by GWAS and selective genotyping were chosen for differential expression analysis to infer the functional regulatory patterns of these genes governing PGH traits in chickpea. The differential expression analysis of PGH trait-associated genes in multiple vegetative and reproductive tissues of diverse (E/SE and $\mathrm{P} / \mathrm{SS} / \mathrm{S}$ ) $\mathrm{PGH}$-bearing chickpea germplasm accessions and RIL mapping individuals were performed (Table S3, Fig. 4). Five candidate genes validated by GWAS and selective genotyping revealed SAM, inflorescence and floral meristems-specific expression ( $>5$-fold) compared with vegetative leaf and root tissues of E/SE and S/SS/P PGH-bearing parents, homozygous mapping individuals and germplasm accessions of chickpea. All these five genes exhibited pronounced differential upregulation ( $>7$-fold, $P \leq 0.001$ ) in $\mathrm{SAM}$, inflorescence and floral meristems of E/SE as compared to that of S/SS/P PGH-bearing individuals/accessions (Fig. 4). Interestingly, these five meristem-specific differentially expressed genes exhibiting significant association with diverse PGH traits (validated by GWAS and selective genotyping) mostly contained non-synonymous coding SNPs. Remarkably, two strong PGH-associated non-synonymous SNPs-containing genes (encoding major intrinsic protein and SNF2 protein) revealed pronounced upregulation ( $>10$-fold, $P \leq 0.0001$ ) in SAM, inflorescence and floral meristems of E/SE than that of S/SS/P PGH-bearing individuals/ accessions of chickpea (Fig. 4). Therefore, it would be interesting to correlate these non-synonymous novel SNP allelic variants mined from different sequence components of the genes with diverse PGH trait association potential and differential expression profiles underlying plant growth habit trait regulation in chickpea. Altogether, five potential differentially expressed genes with non-synonymous functional SNP allelic variants governing PGH traits were delineated in our study by integrating GWAS with selective genotyping and differential expression profiling in natural and mapping populations of chickpea.

The meristem-specific expression, including enhanced upregulation and transcript accumulation of five PGHassociated candidate genes (encoding major intrinsic protein, ankyrin repeat domain containing protein, 


\begin{tabular}{|c|c|c|}
\hline CaKSNP12117-SNF2-ICC13523 & FATATGGGTCAGATTTTGAAGAAAATATGTGATCATCCA & \multirow{14}{*}{$\begin{array}{c}\text { E/SE } \\
\text { accessions, } \\
\text { mapping } \\
\text { parents and } \\
\text { homozygous } \\
\text { RIL } \\
\text { individuals }\end{array}$} \\
\hline & 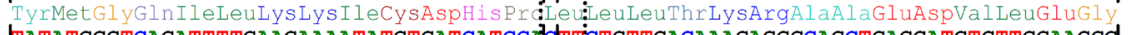 & \\
\hline CaKSNP12117-SNF2-ICC4958 & 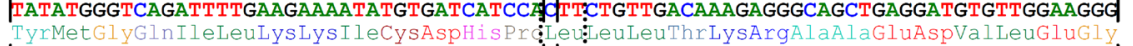 & \\
\hline CaKSNP12117-SNF2-ICC16814 & 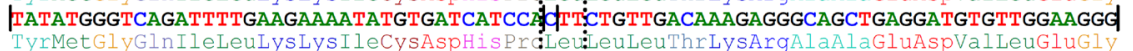 & \\
\hline CaKSNP12117-SNF2-ICC6306 & FATATGGGTCAGATTTTGAAGAAAATATGTGATCATCCAdTTCTGTTGACAAAGAGGGCAGCTGAGGATGTGTTGGAAGG & \\
\hline CaKSNP12117-SNF2-ICC7323 & 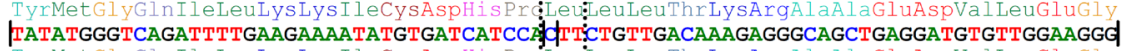 & \\
\hline & 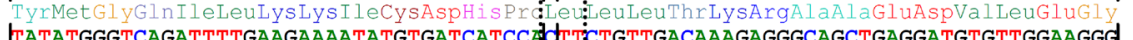 & \\
\hline CaKSNP12117-SNE2-ICC11284 & 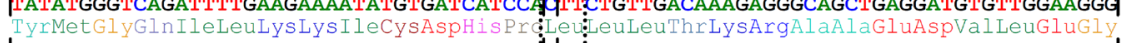 & \\
\hline CaKSNP12117-SNF2-ICC1161 & FATATGGGTCAGATTTTGAAGAAAATATGTGATCATCCA & \\
\hline CaKSNP12117-SNE2-ICC15061 & |FATATGGGTCAGATTITGAAGAAAATATGTGATCATCC & \\
\hline & 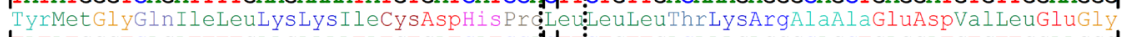 & \\
\hline CaKSNP12117-SNF2-ICC8261 & 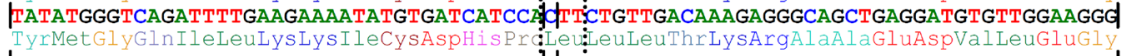 & \\
\hline CaKSNP12117-SNF2-HEM & 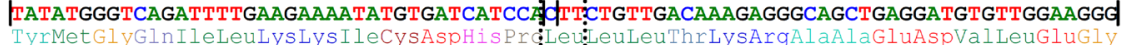 & \\
\hline CaKSNP12117-SNF2-HSEM & |FATATGGGTCAGATTTTGAAGAAAATATGTGATCATCCAd|TCTGTTGACAAAGAGGGCAGCTGAGGATGTGTTGGAAGG & \\
\hline \multirow{2}{*}{ CaKSNP12117-SNE2-ICC12299 } & HATATGGGTCAGATTTTAAG & \\
\hline & yGlnIleLeuLys ysI leCysAspHisPr Ph euLeuThrLysArgAlaAl & \\
\hline CaKSNP12117-SNF2-ICC5590 & 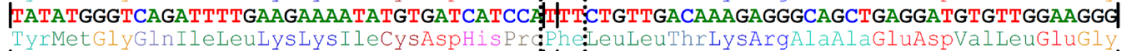 & \\
\hline CaKSNP12117-SNF2-ICC1 & FATATGGGTCAGATTTTGAAGAAAATATGTGATCATCCA ITTCTGTTGACAAAGAGGGCAGCTGAGGATGTGTTGGAAGG & \\
\hline & & \\
\hline aKSNP12117-SNE2-ICC11584 & FATATGGGTCAGATTTTGAAGAAAATATGTGATCATCCAH II TYCTGTTGACAAAGAGGGCAGCTGAGGATGTGTTGGAAGG & \\
\hline CaKSNP12117-SNF2-ICC1164 & 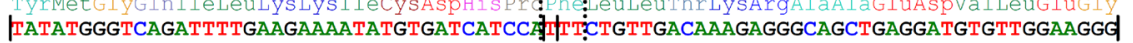 & \\
\hline & 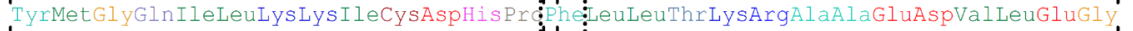 & \\
\hline CaKSNP12117-SNF2-ICC13044 & 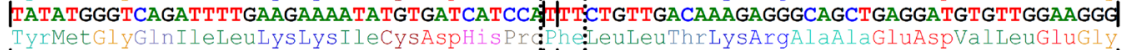 & \\
\hline CaKSNP12117-SNF2-ICC15888 & 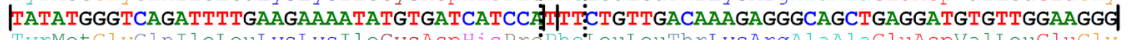 & \\
\hline CaKSNP12117-SNF2-HSM & TCAGATTTTGAA & \\
\hline & G]nTleLeuLystyst leCysAspHisPi & \\
\hline CaKSNP12117-SNF2-HSSM & 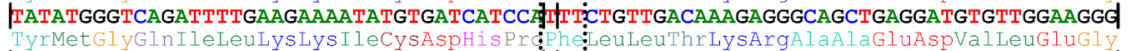 & parents and \\
\hline CaKSNP12117-SNF2-HPM & 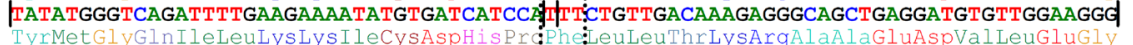 & \\
\hline CaKSNP1211 & 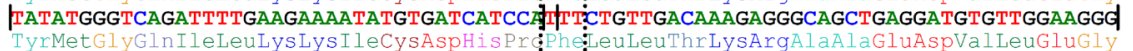 & individuals \\
\hline CaKSNP12117-SNF2-Ce & 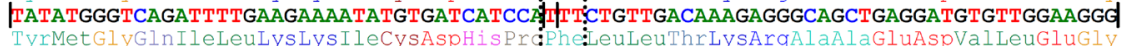 & \\
\hline CaKSNP12117-SNF2-Cj & PATATGGGTCAGATTTTGAAGAAATATGTGATCATCCAMPCTGTTGACAAGAGGGCAGCTGAGGATGTGTTGGAAGG & \\
\hline CaKSNP12117-SNF2-Cb & FATATGGGTCAGATTTTGAAGAAAATATGTGATCATCCAITICTGTTGACAAAGAGGGCAGCTGAGGATGTGTTGG & \\
\hline & 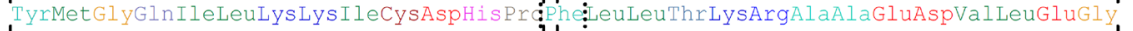 & \\
\hline SNP12117-SNF2-Cp & 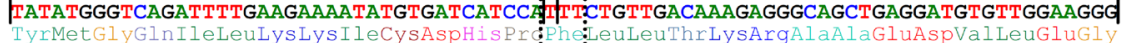 & \\
\hline CaKSNP12117-SNE2-Cm & & \\
\hline & & \\
\hline
\end{tabular}

Fig. 2 The PGH-associated SNP allelic variants (C/T) exhibiting missense non-synonymous amino acid substitutions [(leucine (CTT) to phenylalanine (TTT)] in a vernalization-responsive candidate gene (encoding SNF2 protein) differentiated the E/SE from S/SS/P PGHbearing mapping parents, homozygous RIL individuals and cultivated/ wild accessions of chickpea by selective genotyping. The sequence regions carrying the non-synonymous SNPs are highlighted with dotted boxes. The details regarding the validated SNP loci are mentioned in Tables 1 and S3. HEM/HSEM/HSM/HSSM/HPM: homozygous erect/ semi-erect/spreading/semi-spreading/prostrate mapping individuals, $\mathrm{Cr}$ Cicer reticulatum, Ce C. echinospermum, $C j \mathrm{C}$. judaicum, $\mathrm{Cb} C$. bijugum, $\mathrm{Cp}$ C. pinnatifidum and $\mathrm{Cm}$ C. microphyllum
$\mathrm{ABC}$ transporter, sucrose non-fermenting protein and $\mathrm{B} 3$ transcription factor) scaled down in the present study have ascertained their significant role in controlling growth and development of multiple crop plants. The transcriptional regulation, including differential accumulation of transcripts of these genes in the SAM, inflorescence and floral meristems and their interactions with multiple genes particularly governing the biosynthesis, accumulation, transport and signalling of hormones during vegetative and reproductive growth have been well understood in diverse crop plants, including legumes. The gene encoding plasma membrane major intrinsic proteins (MIPs) is known to be an essential component in controlling major aspects of photosynthesis and plant growth/development (Kelly et al. 2014). The gene encoding ankyrin repeat domain-containing protein plays a key role in plant morphogenesis and architecture by regulating gene expression and modulating meristematic activity of SAM in crop plants (Sharma and Pandey 2016). The transcriptional regulation and function of ankyrin repeat domain-containing protein in influencing plant growth and development has also been elucidated by several previous studies (Nodzon et al. 2004). One of the vernalization-responsive sucrose non-fermenting (SNF2) protein-coding gene plays dynamic role in reduced growth habit without altering other morphological features by its transcript accumulation and chromatin remodelling/modification including maintenance of SAM in multiple plant species (Wang and $\mathrm{Li}$ 2008; Ryan and Owen-Hughes 2011; Sacharowski et al. 2015; Folta et al. 2016). A SNF2 chromatinremodelling protein-coding gene also controls 


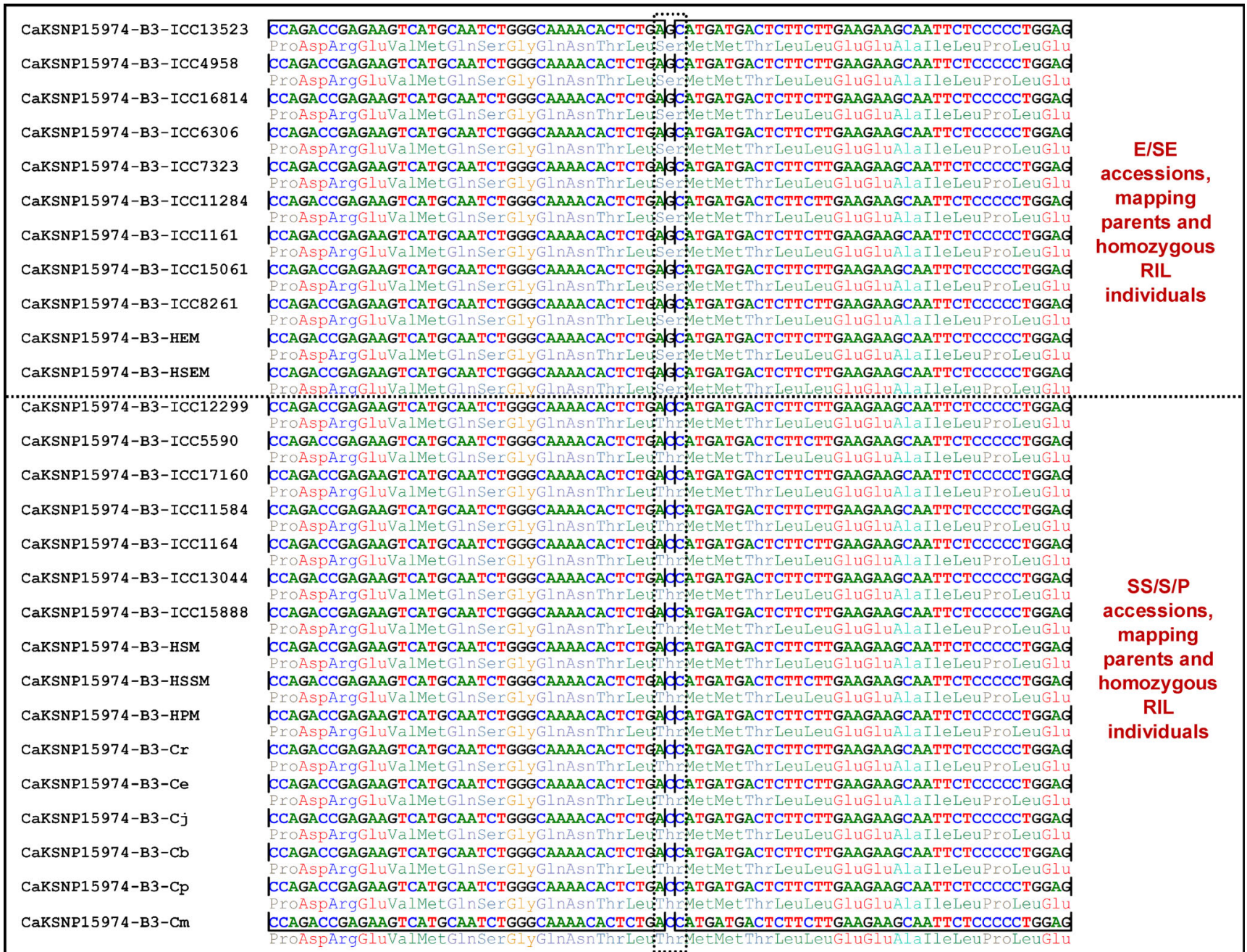

Fig. 3 The PGH-associated SNP allelic variants (G/C) exhibiting missense non-synonymous amino acid substitutions [serine (AGC) to threonine (ACC)] in a vernalization-responsive candidate gene (encoding B3 transcription factor) differentiated the E/SE from S/SS/P PGH-bearing mapping parents, homozygous RIL individuals and cultivated/wild accessions of chickpea by selective genotyping. The sequence regions carrying the non-synonymous SNPs are highlighted

expression and activation of key flowering genes by directly binding to proximal promoter regions of these genes. A $S N F 2$ gene is thus reportedly involved in regulation of both vernalization/flowering responses as well as inflorescence architecture-based plant growth habit trait variation in crop plants. The gene encoding ATPbinding cassette (ABC) transporter underlying a previously reported known PGH major QTLs (mapped on chromosome 3 ) plays a major role in steering phytohormones to integrate endogenous and exogenous signals and thereby assists in synchronizing plant growth with environmental and developmental changes (Cobos et al. 2009; Aryamanesh et al. 2010; Kang et al. 2011; Borghi et al. 2015). The B3 transcription factor family encompassing several distinct gene families, including with dotted boxes. The details regarding the validated SNP loci are mentioned in the Tables 1 and S3. HEM/HSEM/HSM/HSSM/HPM: homozygous erect/semi-erect/spreading/semi-spreading/prostrate mapping individuals, $\mathrm{Cr}$ Cicer reticulatum, $C e \mathrm{C}$. echinospermum, $C j$ C. judaicum, $\mathrm{Cb} C$. bijugum, $\mathrm{Cp} C$. pinnatifidum and $\mathrm{Cm} C$. microphyllum

the LAV [leafy cotyledon2 (LEC2)-abscisic acid insensitive3 (ABI3)-VAL], ARF (auxin response factor), RAV [related to ABI3 and VP1 (viviparous1)] and REM (reproductive meristem) families like VRN 1 (vernalization1) gene, are known to be involved in growth and development as well as flowering and vernalization responses in crop plants (Agarwal et al. 2011). The accumulation of transcripts of DNA ligaseencoding gene is known to enhance plant growth and development (Waterworth et al. 2010). The aforementioned previous reports altogether suggest that the novel functionally relevant natural allelic variants regulating PGH traits scaled down by an integrated genomic approach (GWAS, selective genotyping and expression profiling) can be deployed for rapid quantitative 
Fig. 4 Hierarchical cluster display illustrating the differential expression profiles of seven PGH-associated genes (validated by association analysis and selective genotyping) in vegetative and reproductive tissues of multiple germplasm accessions and homozygous RIL mapping individuals contrasting with diverse PGH traits

(Table S2) using quantitative RTPCR assay. The colour scale at the top represents the average log signal expression values of genes in different tissues; in which green, black and red colour denote low, medium and high level of expression, respectively. The details regarding SNPs and genes are mentioned in the Tables 1 and S3. The tissues and genes used for expression profiling are mentioned on the right and top side of expression map, respectively. The genes exhibiting shoot apical meristem (SAM), inflorescence meristem (IM) and floral meristem (FM)specific expression and differentially upregulated especially in E/SE PGH-bearing germplasm accessions, mapping parents and homozygous RIL individuals are indicated with a red colour box. The HEM/ HSEM/HSM/HSSM/HPM: homozygous erect/semi-erect/ spreading/semi-spreading/ prostrate mapping individuals

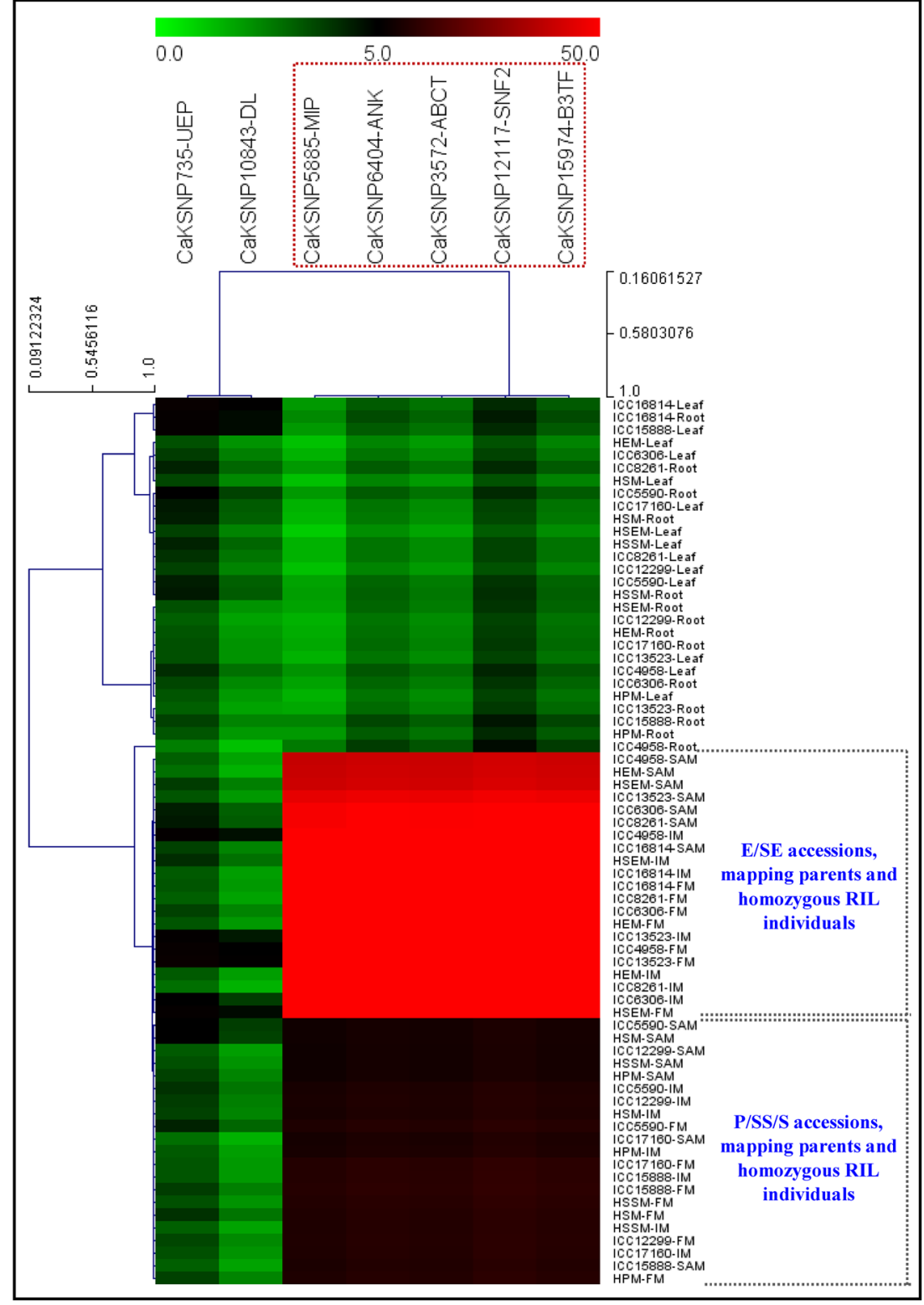

dissection of complex plant growth habit traits in chickpea. These informative molecular tags thus have potential to accelerate marker-assisted genetic enhancement for developing genetically tailored chickpea cultivars with ideal plant architecture of erect growth habit.

\section{Understanding the natural/functional allelic diversity-based selection and domestication pattern of PGH-associated candidate genes}

To infer the natural/functional allelic diversity-led selection and domestication pattern of PGH traits, five PGHassociated genes validated by GWAS, selective genotyping and expression profiling were selected. The PCR amplicons of $100 \mathrm{bp}$ flanking gene regions of these associated SNPs were sequenced among 79 wild chickpea accessions (representing primary, secondary and tertiary gene pools) and compared that with 92 cultivated desi and kabuli chickpea accessions (used for GWAS) for mining natural SNP allelic variants. This exhibited the presence of identical PGH-associated SNP alleles in the five genes which were polymorphic among the E/SE and S/SS PGH-bearing accessions belonging to desi and kabuli cultivar groups as well as 79 prostrate PGH-bearing wild chickpea accessions.

The PGH-associated five SNPs-containing genes were selected to compare and deduce the nucleotide diversity measures as well as the direction and magnitude of natural selection acting on these gene loci across cultivated and wild 
accessions during chickpea domestication. These genes exhibited almost a similar trend of nucleotide diversity $(\theta \pi$ and $\theta \omega)$ and Tajima's D-based selection occurring on SE/E/S/SS PGHbearing 92 desi and kabuli chickpea accessions. The extreme reduction of natural nucleotide diversity level $(\theta \pi$ : 0.41-0.67 and $\theta \omega: 0.47-0.63)$ specifically in SE/E/S/SS PGH-bearing cultivated desi and kabuli chickpea accessions as compared to P PGH-bearing annual/perennial wild accessions $(\theta \pi: 2.57-$ 3.14, $\theta \omega:$ 2.87-3.29) was evident. The reduced nucleotide diversity in cultivated accessions was further supported well with enhanced Tajima's D (3.15 to 4.57) and strong positive selection than that of wild species/accessions encompassing primary, secondary and tertiary gene pools. These findings clearly provide definite evidence regarding signature of strong positive selection on five PGH-associated gene-derived SNP loci in SE/E/S/SS PGH-bearing desi and kabuli chickpea accessions in contrast to prostrate PGH-bearing wild accessions during chickpea domestication. This is agreed well with enduring positive selection for diverse plant architecture contributing traits like plant growth habit suggesting its significance as a target trait for both domestication and artificial breeding in crop plants, including chickpea (Wang and Li 2008; Meyer et al. 2012). The detailed analysis involving the entire natural allelic variants/potential locus targeted by positive selection in PGH-associated genes by constituting molecular haplotypes and selective sweep mapping is required to gain a deeper insight into the complex plant growth habit trait evolution and domestication in chickpea. This will be further useful to decipher the molecular genetic mechanism underlying fixation of such complex growth habit plant architectural quantitative traits especially in domesticated E/SE/S/SS desi and kabuli chickpea accessions that are adapted to diverse agroclimatic conditions as compared to their prostrate PGHbearing wild progenitors.

Vernalization is a crucial key response of plant growth habit and flowering time which has been considered the most vital evolutionary bottleneck during chickpea domestication. This has led to evolution of presently cultivated vernalization insensitive E/SE PGH-bearing desi and kabuli accessions especially from the vernalization sensitive P PGH-bearing wild progenitor C. reticulatum (Abbo et al. 2014). This implicates that $\mathrm{PGH}$ is a major adaptive domestication trait targeted for selective breeding of chickpea accessions growing in diverse agroclimatic conditions. Interestingly, the presence of both SNP allelic variants mined from two vernalizationresponsive candidate genes [encoding SNF2 protein $(\mathrm{C} / \mathrm{T})$ and B3 transcription factor $(\mathrm{G} / \mathrm{C})$ ] associated significantly with $\mathrm{E} / \mathrm{SE}$ vs. S/SS/P PGH trait differentiation was observed in $92 \mathrm{E} / \mathrm{SE}$ and S/SS PGH-bearing cultivated desi and kabuli accessions. In contrast, the occurrence of only the vernalization-responsive $\mathrm{S} / \mathrm{SS} / \mathrm{P}$ PGH trait-associated gene [encoding SNF2 protein (T) and B3 transcription factor (C)]derived SNP alleles was observed in 79 wild accessions representing primary, secondary and tertiary gene pools. The nucleotide diversity $(\theta \pi$ and $\theta \omega)$ and Tajima's D estimation further inferred the influence of very strong positive selection (with enhanced D) in favour of retention of E/SE-associated 'C' SNP in the vernalization responsive SNF2 protein-coding gene specifically in desi and kabuli accessions towards assortment of more preferential E/SE PGH traits in chickpea. Similarly, the preferential selection in favour of retention of E/SE-associated ' $G$ ' SNP in the vernalization responsive B3 transcription factor gene especially in E/SE PGH-bearing desi and kabuli accessions was evident. These findings may be a result of extensive contribution of four sequential bottlenecks (including vernalization) coupled with artificial selection/ modern breeding efforts that are constantly practiced during the genetic improvement program of chickpea for developing cultivars with desirable erect plant growth habit of high preference (Jain et al. 2013; Kujur et al. 2013; Varshney et al. 2013; Abbo et al. 2014; Saxena et al. 2014a, b). These findings infer that the natural SNP allelic variants discovered in the two vernalization-responsive genes are possibly associated with plant growth habit trait evolution, and therefore, the growth habit is expected to represent an important component of domestication trait in chickpea. Therefore, it would be interesting to target these potential genes for mining novel functional allelic variants and haplotypes towards understanding the natural adaptation-based plant growth habit trait evolution and domestication pattern in cultivated and wild chickpea.

Acknowledgments The authors gratefully acknowledge the financial support for this study provided by a research grant from the Department of Biotechnology (DBT), Government of India (102/IFD/SAN/2161/ 2013-14).

\section{References}

Abbo S, van-Oss Pinhasi R, Gopher A, Saranga Y, Ofner I, Peleg Z (2014) Plant domestication versus crop evolution: a conceptual framework for cereals and grain legumes. Trends Plant Sci 19: $351-360$

Agarwal P, Kapoor S, Tyagi AK (2011) Transcription factors regulating the progression of monocot and dicot seed development. BioEssays 33:189-202

Akpinar BA, Lucas S, Budak H (2017) A large-scale chromosome-specific SNP discovery guideline. Funct Integr Genomics 17:97-105

Aryamanesh N, Nelson MN, Yan G, Clarke HJ, Siddique KHM (2010) Mapping a major gene for growth habit and QTLs for Ascochyta blight resistance and flowering time in a population between chickpea and Cicer reticulatum. Euphytica 173:307-319

Bajaj D, Upadhyaya HD, Khan Y, Das S, Badoni S, Shree T, Kumar V, Tripathi S, Gowda CLL, Singh S, Sharma S, Tyagi AK, Chattopdhyay D, Parida SK (2015a) A combinatorial approach of comprehensive QTL-based comparative genome mapping and transcript profiling identified a seed weight-regulating candidate gene in chickpea. Sci Rep 5:9264

Bajaj D, Das S, Upadhyaya HD, Ranjan R, Badoni S, Kumar V, Tripathi S, Gowda CLL, Sharma S, Singh S, Tyagi AK, Parida SK (2015b) A 
genome-wide combinatorial strategy dissects complex genetic architecture of seed coat color in chickpea. Front Plant Sci 6:979

Bajaj D, Saxena MS, Kujur A, Das S, Badoni S, Tripathi S, Upadhyaya HD, Gowda CLL, Sharma S, Singh S, Tyagi AK, Parida SK (2015c) Genome-wide conserved non-coding microsatellite (CNMS) marker-based integrative genetical genomics for quantitative dissection of seed weight in chickpea. J Exp Bot 66:1271-1290

Bajaj D, Upadhyaya HD, Das S, Kumar V, Gowda CLL, Sharma S, Tyagi AK, Parida SK (2016) Identification of candidate genes for dissecting complex branch number trait in chickpea. Plant Sci 245:61-70

Benjamini Y, Hochberg Y (1995) Controlling the false discovery rate: a practical and powerful approach to multiple testing. J R Stat Soc B 57:289-300

Benlloch R, Berbel A, Ali L, Gohari G, Millán T, Madueño F (2015) Genetic control of inflorescence architecture in legumes. Front Plant Sci 6:543

Borghi L, Kang J, Ko D, Lee Y, Martinoia E (2015) The role of ABCGtype $\mathrm{ABC}$ transporters in phytohormone transport. Biochem Soc Trans 43:924-930

Cobos MJ, Winter P, Kharrat M, Cubero JI, Gil J, Milian T, Rubio J (2009) Genetic analysis of agronomic traits in a wide cross of chickpea. Field Crops Res 111:130-136

Das S, Upadhyaya HD, Bajaj D, Kujur A, Badoni S, Laxmi, Kumar V, Tripathi S, Gowda CLL, Sharma S, Singh S, Tyagi AK, Parida SK (2015) Deploying QTL-seq for rapid delineation of a potential candidate gene underlying major trait-associated QTL in chickpea. DNA Res 22:193-203

Das S, Singh M, Srivastava R, Bajaj D, Saxena MS, Rana JC, Bansal KC, Tyagi AK, Parida SK (2016) mQTL-seq delineates functionally relevant candidate gene harbouring a major QTL regulating pod number in chickpea. DNA Res 23:53-65

Feuillet C, Stein N, Rossini L, Praud S, Mayer K, Schulman A, Eversole $\mathrm{K}$, Appels R (2012) Integrating cereal genomics to support innovation in the Triticeae. Funct Integr Genomics 12:573-583

Folta A, Bargsten JW, Bisseling T, Nap JP, Mlynarova L (2016) Compact tomato seedlings and plants upon overexpression of a tomato chromatin remodelling ATPase gene. Plant Biotechnol J 14:581-591

Gaur R, Azam S, Jeena G, Khan AW, Choudhary S, Jain M, Yadav G, Tyagi AK, Chattopadhyay D, Bhatia S (2012) High-throughput SNP discovery and genotyping for constructing a saturated linkage map of chickpea (Cicer arietinum L.) DNA Res 19:357-373

Gupta S, Nawaz K, Parween S, Roy R, Sahu K, Pole AK, Khandal H, Srivastava R, Parida SK, Chattopadhyay D (2016) Draft genome sequence of Cicer reticulatum L., the wild progenitor of chickpea provides a resource for agronomic trait improvement. DNA Res 24: $1-10$

Jain M, Misra G, Patel RK, Priya P, Jhanwar S, Khan AW, Shah N, Singh VK, Garg R, Jeena G, Yadav M, Kant C, Sharma P, Yadav G, Bhatia S, Tyagi AK, Chattopadhyay D (2013) A draft genome sequence of the pulse crop chickpea (Cicer arietinum L.) Plant J 74:715-729

Kang J, Park J, Choi H, Burla B, Kretzschmar T, Lee Y, Martinoia E (2011) Plant ABC transporters. Arabidopsis book 9:e0153

Kelly G, Sade N, Attia Z, Secchi F, Zwieniecki M, Holbrook NM, Levi A, Alchanatis V, Moshelion M, Granot D (2014) Relationship between hexokinase and the aquaporin PIP1 in the regulation of photosynthesis and plant growth. PLoS One 9:e87888

Kujur A, Bajaj D, Saxena MS, Tripathi S, Upadhyaya HD, Gowda CLL, Singh S, Jain M, Tyagi AK, Parida SK (2013) Functionally relevant microsatellite markers from chickpea transcription factor genes for efficient genotyping applications and trait association mapping. DNA Res 20:355-374

Kujur A, Bajaj D, Saxena MS, Tripathi S, Upadhyaya HD, Gowda CLL, Singh S, Tyagi AK, Jain M, Parida SK (2014) An efficient and costeffective approach for genic microsatellite marker-based large-scale trait association mapping: identification of candidate genes for seed weight in chickpea. Mol Breed 34:241-265
Kujur A, Bajaj D, Upadhyaya HD, Das S, Ranjan R, Shree T, Saxena MS, Badoni S, Kumar V, Tripathi S, Gowda CLL, Sharma S, Singh S, Tyagi AK, Parida SK (2015a) A genome-wide SNP scan accelerates trait-regulatory genomic loci identification in chickpea. Sci Rep 5: 11166

Kujur A, Bajaj D, Upadhyaya HD, Das S, Ranjan R, Shree T, Saxena MS, Badoni S, Kumar V, Tripathi S, Gowda CLL, Sharma S, Singh S, Tyagi AK, Parida SK (2015b) Employing genome-wide SNP discovery and genotyping strategy to extrapolate the natural allelic diversity and domestication patterns in chickpea. Front Plant Sci 6: 162

Kujur A, Upadhyaya HD, Shree T, Bajaj D, Das S, Saxena M, Badoni S, Kumar V, Tripathy S, Gowda CLL, Sharma S, Singh S, Tyagi AK, Parida SK (2015c) Ultra-high density intra-specific genetic linkage maps accelerate identification of functionally relevant molecular tags governing important agronomic traits in chickpea. Sci Rep 5: 9468

Kumar A, Choudhary AK, Solanki RK, Pratap A (2011) Towards marker-assisted selection in pulses: a review. Plant breed 130:297313

Lipka AE, Tian F, Wang Q, Peiffer J, Li M, Bradbury PJ, Gore MA, Buckler ES, Zhang Z (2012) GAPIT: genome association and prediction integrated tool. Bioinformatics 2:2397-2399

Liu Z, Zhu C, Jiang Y, Tian Y, Yu J, An H, Tang W, Sun J, Tang J, Chen G, Zhai H, Wang C, Wan J (2016) Association mapping and genetic dissection of nitrogen use efficiency-related traits in rice (Oryza sativa L.) Funct Integr Genomics 16:323-333

Meyer RS, DuVal AE, Jensen HR (2012) Patterns and processes in crop domestication: an historical review and quantitative analysis of 203 global food crops. New Phytol 196:29-48

Nodzon LA, Xu WH, Wang Y, Pi LY, Chakrabarty PK, Song WY (2004) The ubiquitin ligase XBAT32 regulates lateral root development in Arabidopsis. Plant J 40:996-1006

Parween S, Nawaz K, Roy R, Pole AK, Venkata Suresh B, Misra G, Jain M, Yadav G, Parida SK, Tyagi AK, Bhatia S, Chattopadhyay D (2015) An advanced draft genome assembly of a desi type chickpea (Cicer arietinum L.) Sci Rep 5:12806

Ryan DP, Owen-Hughes T (2011) Snf2-family proteins: chromatin remodellers for any occasion. Curr Opin Chem Biol 15:649-656

Sacharowski SP, Gratkowska DM, Sarnowska EA, Kondrak P, Jancewicz I, Porri A, Bucior E, Rolicka AT, Franzen R, Kowalczyk J, Pawlikowska K, Huettel B, Torti S, Schmelzer E, Coupland G, Jerzmanowski A, Koncz C, Sarnowski TJ (2015) SWP73 subunits of Arabidopsis SWI/SNF chromatin remodeling complexes play distinct roles in leaf and flower development. Plant Cell 27:18891906

Saxena MS, Bajaj D, Kujur A, Das S, Badoni S, Kumar V, Singh M, Bansal KC, Tyagi AK, Parida SK (2014a) Natural allelic diversity, genetic structure and linkage disequilibrium pattern in wild chickpea. PLoS One 9:e107484

Saxena MS, Bajaj D, Das S, Kujur A, Kumar V, Singh M, Bansal KC, Tyagi AK, Parida SK (2014b) An integrated genomic approach for rapid delineation of candidate genes regulating agro-morphological traits in chickpea. DNA Res 21:695-710

Sharma M, Pandey GK (2016) Expansion and function of repeat domain proteins during stress and development in plants. Front Plant Sci 6: 1218

Thudi M, Upadhyaya HD, Rathore A, Gaur PM, Krishnamurthy L, Roorkiwal M, Nayak SN, Chaturvedi SK, Basu PS, Gangarao NV, Fikre A, Kimurto P, Sharma PC, Sheshashayee MS, Tobita S, Kashiwagi J, Ito O, Killian A, Varshney RK (2014) Genetic dissection of drought and heat tolerance in chickpea through genome-wide and candidate gene-based association mapping approaches. PLoS One 9:e96758

Upadhyaya HD, Furman BJ, Dwivedi SL, Udupaa SM, Gowdaa CLL, Bauma M, Croucha JH, Buhariwallaa HK, Singh S (2006) 
Development of a composite collection for mining germplasm possessing allelic variation for beneficial traits in chickpea. Plant Genet Resour 4:13-19

Upadhyaya HD, Dwivedi SL, Baum M, Varshney RK, Udupa SM, Gowda CLL, Hoisington D, Singh S (2008) Genetic structure, diversity and allelic richness in composite collection and reference set in chickpea (Cicer arietinum L.) BMC Plant Biol 8:106

Upadhyaya HD, Bajaj D, Das S, Saxena MS, Badoni S, Kumar V, Tripathi S, Sharma S, Tyagi AK, Parida SK (2015) A genomescale integrated approach aids in genetic dissection of complex flowering time trait in chickpea. Plant Mol Biol 89:403-420

Upadhyaya HD, Bajaj D, Narnoliya L, Das S, Kumar V, Gowda CLL, Sharma S, Tyagi AK, Parida SK (2016) Genome-wide scans for delineation of candidate genes regulating seed-protein content in chickpea. Front Plant Sci 7:302

Varshney RK, Song C, Saxena RK et al. (2013) Draft genome sequence of chickpea (Cicer arietinum) provides a resource for trait improvement. Nat Biotechnol 31:240-246
Varshney RK, Mir RR, Bhatia S, Thudi M, Hu Y, Azam S, Zhang Y, Jaganathan D, You FM, Gao J, Riera-Lizarazu O, Luo MC (2014) Integrated physical, genetic and genome map of chickpea (Cicer arietinum L.) Funct Integr Genomics 14:59-73

Wang Y, Li J, (2008) Molecular basis of plant architecture. Annu Rev Plant Biol 59(1):253-279

Waterworth WM, Masnavi G, Bhardwaj RM, Jiang Q, Bray CM, West CE (2010) A plant DNA ligase is an important determinant of seed longevity. Plant J 63:848-860

Xu X, Liu X, Ge S, Jensen JD, Hu F, Li X, Dong Y, Gutenkunst RN, Fang L, Huang L, Li J, He W, Zhang G, Zheng X, Zhang F, Li Y, Yu C, Kristiansen K, Zhang X, Wang J, Wright M, McCouch S, Nielsen R, Wang J, Wang W (2011) Resequencing 50 accessions of cultivated and wild rice yields markers for identifying agronomically important genes. Nat Biotechnol 30:105-111

Zhang Z, Ersoz E, Lai CQ, Todhunter RJ, Tiwari HK, Gore MA, Bradbury PJ, Yu J, Arnett DK, Ordovas JM, Buckler ES (2010) Mixed linear model approach adapted for genome-wide association studies. Nat Genet 42:355-368 